Proceedings of the WELCOME Scientific Meeting on Hybrid Nanostructures, Toruń, Poland, August 28-31, 2011

\title{
Influence of Plasmon Resonance in Silver Island Film on the Optical Properties of Peridinin-Chlorophyll-Protein Light-Harvesting Complexes
}

\author{
K.K. Ciszak ${ }^{a, *}$, M. Olejnik ${ }^{a}$, J. Strzelecki ${ }^{a}$, B. Krajnik ${ }^{a}$, D. Piątkowski ${ }^{a}$, \\ E. HOFMANN ${ }^{b}$ AND S. MACKOWSKI ${ }^{a}$ \\ ${ }^{a}$ Institute of Physics, Nicolaus Copernicus University, Grudziądzka 5, 87-100 Toruń, Poland \\ ${ }^{b}$ Department of Biology and Biotechnology, Ruhr-University Bochum, D-44780 Bochum, Germany \\ We study the effect of plasmon excitations in silver island film on the optical properties of peridinin- \\ chlorophyll-protein light-harvesting complex using scanning fluorescence microscopy. With this technique we can \\ unambiguously locate areas where the biomolecules are deposited on the metallic nanostructures from the areas \\ where they stick to the glass surface. The enhancement factor of fluorescence intensity obtained for such a hybrid \\ nanostructure is found to be 3. Plasmon excitations in the SIF layer also influence the dynamics of the emission, \\ but in this case the interpretation of the results is more complex.
}

PACS: 33.50.Dq, 73.20.Mf, 87.15.M-, 81.07.Pr

\section{Introduction}

Plasmon resonance, a characteristic property of metallic nanoparticles, has become in recent years an important tool to modify electromagnetic fields at the nanoscale [1]. The frequency and linewidth of the plasmon resonance depends upon the size and geometry of a metallic nanoparticle; while for spherical nanoparticles the resonances appear around $420 \mathrm{~nm}$ and $530 \mathrm{~nm}$ for silver and gold, respectively, other geometries, such as nanorods or nanoshells feature strong resonances in the infrared spectral region. Thus in order to explore ways the morphology reflects itself in the optical properties of metallic nanoparticles, the range of systems has been investigated, including spherical nanoparticles, nanorods, nanowires, or thin films of nanosized islands. Through tailoring the morphology and spatial arrangement, metallic nanoparticles have been applied to concentrate optical fields [2], direct molecular fluorescence [3], detect local chemical surroundings [4], and enhance the absorption and emission of placed nearby dipoles [5-7]. One of the simplest metallic nanostructure, yet providing efficient ways to tune the optical properties of fluorophores, is a silver island film (SIF) [8]. In addition to being applied to increase the fluorescence radiative rate of simple molecular systems, the SIF substrates have found application in enhancing the absorption of natural light-harvesting complexes [9-11].

The exact outcome of the plasmon induced coupling with fluorescent molecules depends on the spectral properties of both metallic nanoparticles and fluorophores. For systems where the absorption of the SIF coincides

* corresponding author; e-mail: ciszak@fizyka.umk.pl with absorption of the molecule, the enhancement of the absorption rate is expected [9]. Conversely, should the plasmon resonance in the SIF overlap with the emission spectrum, the most dominant effect is associated with an increase of the fluorescence rate [5].

In this work we use confocal fluorescence imaging to study the influence of plasmon excitations in SIF on the fluorescence properties of peridinin-chlorophyllprotein (PCP) isolated from the algae Amphidinium carterae [12]. Applying the imaging to study such a hybrid nanostructure allows for exact determination of areas where the SIF layer was deposited during the synthesis as well as the areas free of the metallic nanoparticles. In this way we can unambiguously attribute spectral changes, measured both with continuous wave and time-resolved spectroscopies to the interaction between the pigment-protein light-harvesting complex and the plasmon excitations in the silver island film. The results show strong increase of the fluorescence intensity for the PCP complexes coupled to the plasmon excitations in the SIF layer.

\section{Materials and methods}

In its native form the PCP complex is a trimer with each monomer containing two chlorophyll $a(\mathrm{Chl} a)$ and eight peridinin (Per) molecules embedded in the protein matrix. The structure of this water-soluble antenna has been determined with $1.5 \AA$ resolution using X-ray crystallography [12] and is shown in Fig. 1a. Four Per around one Chl $a$ form a cluster connected via van der Waals interactions. Per are the major light-harvesting pigment in the PCP and due to close proximity to the Chl $a$ they can transfer energy with the quantum efficiency of over $90 \%$ [13]. The distance between the $\mathrm{Mg}$ atoms of the 
two Chl $a$ is $17.4 \AA$. The optical properties of the PCP complex have been studied in detail, both for ensembles and on the single-molecule level $[14,15]$. These experiments determined the interactions between pigments as well as dominant energy transfer pathways in this complex. In this work we use PCP complexes reconstituted with Chl $a$. The protocols of reconstitution were published previously [16]. Obtained protein was closed in TRIS buffer ( $5 \mathrm{mM}, 7.6 \mathrm{pH})$ and kept frozen until used.
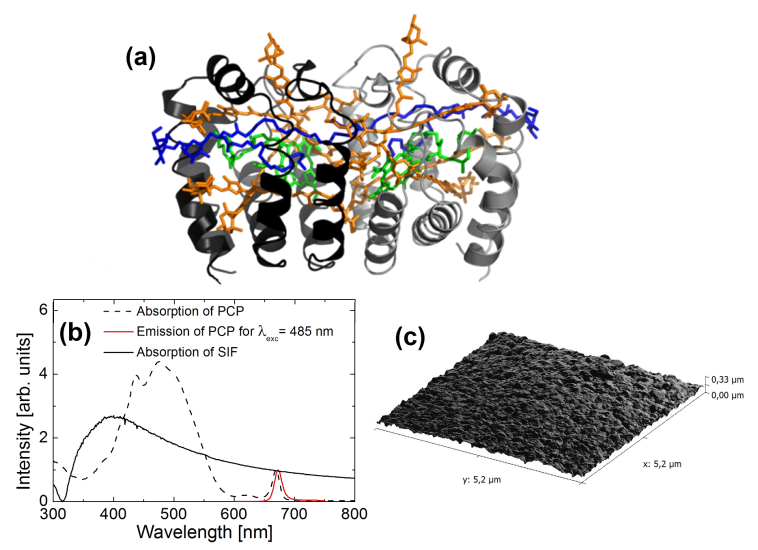

Fig. 1. (a) PCP monomer, which contains two chlorophyll $a$ (green) and eight peridinins (orange) embedded in a protein matrix (gray spiral); (b) comparison of absorption spectra of PCP (dashed line), and the SIF layer (black line) together with the emission spectrum of the PCP complexes (red curve); (c) morphology of the SIF surface imaged using AFM.

SIF was fabricated by reduction of silver nitrate by sodium hydroxide and D-glucose [6, 10]. All chemicals were bought from Sigma-Aldrich and used without further purification. First, we dissolve $375 \mathrm{mg}$ of silver nitrate in $45 \mathrm{ml}$ of water and then add $1.5 \mathrm{ml}$ of $5 \%$ aqueous solution of sodium hydroxide. After that we add $1 \mathrm{ml}$ of $25 \%$ aqueous solution of ammonia. The mixture was then cooled down to $5{ }^{\circ} \mathrm{C}$. Firstly, cover slips was cleaned in special solution named piranha solution which contains one portion of peroxide $\left(\mathrm{H}_{2} \mathrm{O}_{2}\right)$ and three portions of sulfuric acid (VI) $\left(\mathrm{H}_{2} \mathrm{SO}_{4}\right)$. Glass had been kept in this solution for overnight before it was used. Second step was main synthesis. When the temperature reaches $5{ }^{\circ} \mathrm{C}$, $540 \mathrm{mg}$ of D-glucose dissolved in $11 \mathrm{ml}$ of water was added to solution and simultaneously eight cover slips were put into the mixture. After that solution with cover slips had been heated to $30^{\circ} \mathrm{C}$ and was put up after 5 min (time was started count when solution reaches $30^{\circ} \mathrm{C}$ ) and they have been sonicated for one minute. After that all cover slips were put into pure water.

The obtained SIF substrates were analyzed using atomic force microscopy (AFM) technique and absorption spectroscopy to determine plasmon resonance frequency. The morphology was studied using Bioscope II atomic force microscope (Veeco) in a contact mode. Absorption measurements of the SIF substrates shortly after removing them from the reaction solution were acquired at room temperature using Carry $50 \mathrm{UV}-\mathrm{V}$ is spectrophotometer in the spectral range from 350 to $1100 \mathrm{~nm}$. The spectrum obtained for the SIF substrate was adjusted to the clean coverslip.

The hybrid nanostructure comprising the PCP complexes and the SIF was fabricated as follows. First, the stock solution of the PCP complexes $(1.5 \mathrm{mg} / \mathrm{ml})$ was diluted 100 times in $2 \%$ aqueous PVA. Suspending the protein in polymer makes it more stable than in water. Next, $40 \mu$ l of the PCP complexes diluted in PVA were spin coated on SIF surface. The estimated thickness of the PVA layer containing the PCP complexes was about $200 \mathrm{~nm}$.

The optical properties of the fabricated hybrid structures were measured using our confocal scanning fluorescence microscope [17] which allows for collecting fluorescence maps, fluorescence spectra, and the lifetimes. Optics is based on a microscope objective LMPlan $50 \mathrm{x}$ (Olympus) with numerical aperture 0.5 and working distance of $6 \mathrm{~mm}$. The sample is placed on a piezoelectric $X Y Z$ stage (Physik Instrumente) with a single step nominal resolution around $1 \mathrm{~nm}$. The fluorescence map is then obtained by correlating the movement of the piezo stage with the readout of the detector. The sample was excited using a $485 \mathrm{~nm}$ laser that can provide continuous or pulsed excitation. The FWHM of laser pulse is $70 \mathrm{ps}$ and the repetition rate of $50 \mathrm{MHz}$ was used. The average power of the laser was about $100 \mu \mathrm{W}$. The fluorescence is detected in a back-scattering geometry, it is first focused on the pinhole ( $150 \mu$ m diameter $)$ and then guided to any of the three detectors. The intensity of the emission is extracted using two bandpass filters: HQ $600+$ and $\mathrm{HQ}$ 670/10 (Chroma) before it reaches an avalanche photodiode detector PerkinElmer SPCM-AQRH-14 (dark count $80 \mathrm{cps}$ ). Fluorescence lifetimes are extracted using time-correlated single photon counting (TCSPC) technique, through a combination of the SPC-150 card (Becker and Hickl) and fast avalanche photodiode idQuantique id100-50 (dark count $50 \mathrm{cps}$ ). The time resolution is about 50 ps. Finally, the spectra are measured in a configuration, in which a CCD detector (iDus DV 420A-BV) is combined with an Amici prism giving the spectral resolution of about $2 \mathrm{~nm}$ in the spectral region of interest.

\section{Results and discussion}

A typical AFM image is shown in Fig. 1c. The size of silver islands varies between $50 \mathrm{~nm}$ and $200 \mathrm{~nm}$, which is typical for this kind of plasmonic substrate [6]. Such large inhomogeneity results in quite broad absorption band attributed to plasmon resonance in the islands, as displayed in Fig. 1b. The absorption spectrum shows a sharp resonance at the wavelength of $400 \mathrm{~nm}$ with a very broad tail towards longer wavelengths.

Importantly, the absorption spectrum of the SIF sample correlates well with the absorption spectrum of the PCP complexes. Both are compared in Fig. 1b. The PCP 
absorption features broad band from $400 \mathrm{~nm}$ to $550 \mathrm{~nm}$, which is mainly due to peridinin absorption. The contribution of chlorophylls can be found around at wavelengths shorter than $400 \mathrm{~nm}$ and around $660 \mathrm{~nm}$. The emission of PCP complexes originates from the $Q_{y}$ band of the chlorophylls and its maximum is at $673 \mathrm{~nm}$.

Before we discuss the results obtained for the PCP complexes deposited on the SIF substrate, we would like to point out that in addition to the inhomogeneity associated with the size variation of the silver islands, there is also another parameter, which varies over two orders of magnitude. As displayed in Fig. 2, the actual sample structure features PCP complexes at distances anywhere beteween 0 and $200 \mathrm{~nm}$ from the silver islands. As the interaction with plasmon excitations in metallic nanoparticles depends strongly upon the distance, we could expect large variation in observed intensities of fluorescence emission. Such a behavior was indeed observed in a single-molecule experiment [10]. Therefore, when large ensemble is probed, only average values can be extracted.

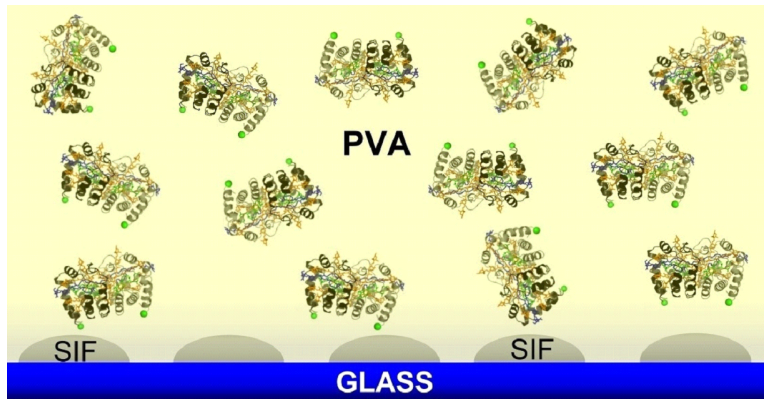

Fig. 2. Schematic cross-section of the sample containing $\mathrm{PCP}$ in a PVA matrix spin-coated on SIF surface.

The fluorescence intensity map measured for PCP complexes deposited onto the SIF substrate is shown in Fig. 3a. The size of the map is $100 \mu \mathrm{m} \times 100 \mu \mathrm{m}$, the step was $500 \mathrm{~nm}$ and the acquisition time for a single point was $0.01 \mathrm{~s}$. Two clearly distinguishable regions can be identified on the map. Dark areas, characterized with average intensities of less than 200 counts are attributed to SIF-less regions of the sample. On the other hand, we also observe high-intensity regions, with average fluorescence intensity of about 600 counts.

In order to demonstrate better the difference between the two areas we extracted intensities of individual pixels, as shown in Fig. 3b. The spread of the intensities observed for the plasmon-enhanced regions is significantly broader than the one corresponding to the glass surface. Such a broadening is expected for plasmonic nanostructures, as the interaction with metallic nanoparticles depends on variety of parameters that introduces additional disorder. From the histogram we determine the average enhancement factor to be around 3-fold.

In Fig. 3c we show the fluorescence spectra measured on the areas marked in Fig. 3a. Both spectra are iden-
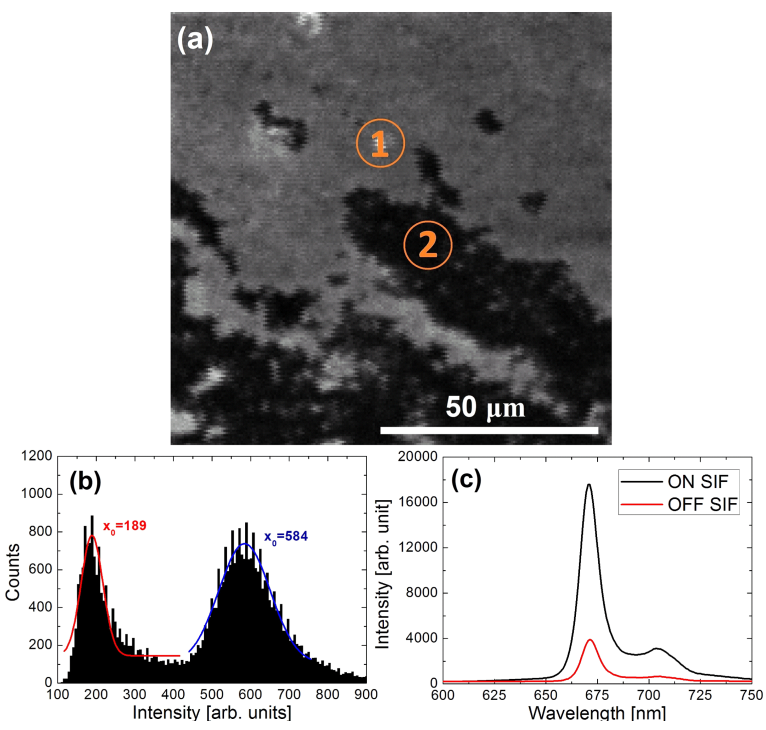

Fig. 3. (a) Confocal fluorescence microscopy map of PCP emission on the SIF surface obtained with laser excitation of $485 \mathrm{~nm}$; (b) histogram of intensity extracted from the map together with two Gaussian functions; (c) fluorescence spectra measured for the PCP complexes placed on (black) and off (red) the SIF layer.

tical in shape, the only difference is the intensity. The absence of any modification of the fluorescence emission upon coupling with plasmon excitations in metallic layer indicates that the protein of the PCP complex remains intact in such a hybrid nanostructure. This observation is vital for any possible consideration of assembling a functional device based on light-harvesting complexes.

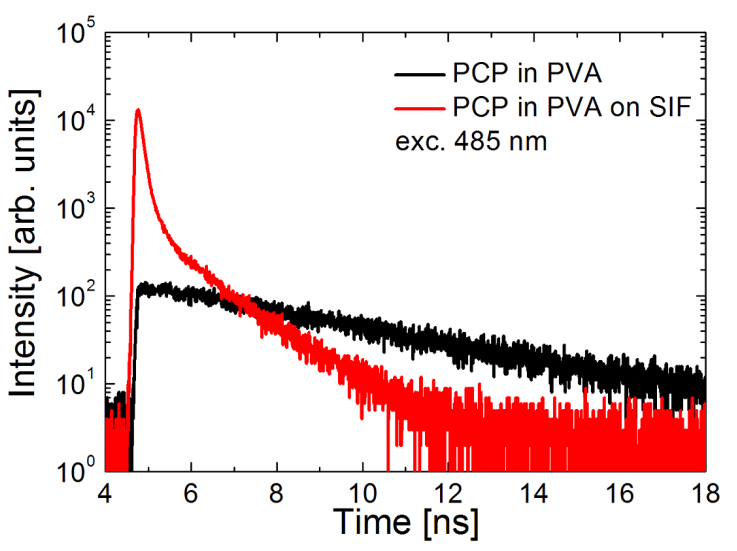

Fig. 4. Fluorescence transients measured for $\mathrm{PCP}$ on glass (black) and PCP on the SIF substrate (red). The excitation energy of $485 \mathrm{~nm}$ was used.

The influence of plasmon excitations upon the optical properties of the light-harvesting complexes can also be observed in the transient behavior of the fluorescence. The results of an experiment carried out for PCP complexes deposited on the SIF substrate and on a glass cov- 
erslip are shown in Fig. 4. The transient measured for the reference sample (black curve) features monoexponential decay characterized with the lifetime of $3.7 \mathrm{~ns}$. This result agrees well with previous measurements [10]. The red curve displays the result measured for the PCP complexes on the SIF surface. The decay for this sample is more complicated, it is at least biexponential. Clearly, the impact of plasmon excitations substantially modifies the fluorescence characteristics of the chlorophylls confined to the PCP complexes. Unfortunately, due to inhomogeneities of the sample it is difficult to associate the lifetimes with particular geometry of PCP arrangement versus the silver island. Indeed, both quenched complexes as well as those where the radiative rate is enhanced would feature much shorter fluorescence lifetimes. In order to solve this issue single molecule experiment in time domain is necessary. Assuming that at least one part of the shortening of the fluorescence lifetime is due to PCP complexes that are efficiently coupled to the silver islands, we can speculate that the plasmon excitation in the SIF layer is somewhat transferred between the islands. It stems from the fact that for time-resolved experiment the excitation wavelength of $485 \mathrm{~nm}$ was used, which clearly excites plasmons in the SIF layer but in the spectral range that is at much higher energies than the PCP emission. Thus, we suggest that these high-energy plasmons can efficiently transfer their energy towards low energy plasmons that are resonant with the emission of the PCP complexes. In this way coupling in the spectral range around $670 \mathrm{~nm}$ becomes possible that could lead to strong modifications of the fluorescence lifetimes observed in the experiment. Further experiments are required in order to resolve this point.

\section{Conclusions}

We demonstrate strong influence of the plasmon excitations in the SIF layer on the optical properties of the PCP light-harvesting complexes. Using confocal fluorescence imaging we can determine precisely areas where the complexes are deposited on the SIF. The intensity of fluorescence measured for coupled PCP complexes is on average 3-fold higher than for the reference pointing towards strong enhancement. The plasmon coupling leads also to pronounced modifications of the fluorescence lifetimes indicating transfer of plasmon excitation within the SIF layer.

\section{Acknowledgments}

Financial support from the WELCOME program "Hybrid nanostructures as a stepping-stone towards efficient artificial photosynthesis" awarded by the Foundation for Polish Science is gratefully acknowledged.

\section{References}

[1] S. Maier, Plasmonics: Fundamentals and Applications, Springer Science + Business Media LLC, New York 2007.

[2] L. Novotny, N. van Hulst, Nature Phot. 5, 83 (2011).

[3] L. Rogobete, F. Kaminski, M. Agio, V. Sandoghdar, Opt. Lett. 32, 1623 (2007).

[4] J. Lee, P. Hernandez, J. Lee, A.O. Govorov, N.A. Kotov, Nature Mater. 6, 291 (2007).

[5] P. Bharadwaj, P. Anger, L. Novotny, Nanotechnology 18, 044017 (2007).

[6] K. Ray, R. Badugu, J.R. Lakowicz, J. Am. Chem. Soc. 128, 8998 (2006).

[7] J. Lee, A.O. Govorov, N.A. Kotov, Nano Lett. 5, 10 (2005).

[8] J.R. Lakowicz, Anal. Biochem. 298, 1 (2001).

[9] N. Czechowski, P. Nyga, M.K. Schmidt, T.H.P. Brotosudarmo, H. Scheer, D. Piatkowski, S. Mackowski, Plasmonics 7, 115 (2012).

[10] S. Mackowski, J. Phys., Condens. Matter 22, 193102 (2010).

[11] J. Nieder, R. Bittl, M. Brecht, Angew. Chem. 49, 10217 (2010)

[12] E. Hofmann, P.M. Wrench, F.P. Sharples, R.G. Hiller, W. Welte, K. Diederichs, Science 272, 5269 (1996).

[13] T. Polivka, V. Sundstrom, Chem. Rev. 104, 2021 (2004).

[14] F.J. Kleima, M. Wendling, E. Hofmann, E.J.G. Peterman, R. van Grondelle, H. van Amerongen, Biophys. J. 78, 344 (2000).

[15] S. Wormke, S. Mackowski, T.H.P. Brotosudarmo, C. Jung, A. Zumbusch, M. Ehrl, H. Scheer, E. Hofmann, R.G. Hiller, C. Brauchle, Biochim. Biophys. Acta 1767, 956 (2007).

[16] T.H.P. Brotosudarmo, E. Hofmann, R.G. Hiller, S. Wormke, S. Mackowski, A. Zumbusch, C. Brauchle, H. Scheer, FEBS Lett. 580, 5257 (2006).

[17] B. Krajnik, T. Schulte, D. Piątkowski, N. Czechowski, E. Hofmann, S. Mackowski, Cent. Eur. J. Phys. 2, 293 (2011). 